\title{
Enterprise Representation: An Analysis of Standards Issues
}

\author{
J. G. Nell, Convener \\ ISO TC184/SC5/WG1, Industrial Automation Systems and Integration: Modeling \\ and Architecture \\ National Institute of Standards and Technology; Bldg. 220, Rm. A127 \\ Gaithersburg MD, USA 20899 \\ $13019755748(V), 13012589749(F)$, nell@cme.nist.gov
}

\begin{abstract}
The purpose of this paper is to describe the domain of standards with respect to achieving the best enterprise integration. There are some definitions about frameworks and architectures, discussions re the use of enterprise models and modeling, the key components and characteristics of these models, a description of the users of the models and their needs, enterprise-model drivers and their relation to enterprise integration, and the nature and advantages of international standards covering enterprise-reference architectures, modeling, and models.

This paper identifies issues that, if resolved, will help define a realistic role for standards in defining the different necessary components of enterprise integration. The representation of the enterprise is, by definition, a model. One could view the enterprise-reference architecture as information and knowledge with which one could adequately represent the enterprise. One could then consider the enterprise-reference architecture to be a meta model of the enterprise representation. The enterprise-architecture is a component of this meta model.

The ensuing standards can help vendors create software products that enable the information transfers required by any organization of processes. Accomplishing these transfers will provide a path for enterprises to approach higher levels of integration by defining guidelines for designing new enterprises that can operate in a more integrated mode, and for creating enterprise models. The domain of these standards can range from standardizing the enterprise to standard names for key concepts. This paper will attempt to point out which of these are relevant material for standardization.
\end{abstract}

\section{Key Words}

Enterprise, integration, architecture, framework, enterprise model, virtual enterprise, infrastructure, process, life cycle, enterprise representation, international standard, terminology. 


\section{ENTERPRISES, PRODUCTS, PROCESSES, AND INTEGRATION}

The manufacturing enterprise is a group of processes that produce a product (an output). An enterprise can be of any size from a multi-company endeavor; for example, to build an airplane, to a single process like soldering. Therefore, an enterprise can be any group of processes of interest at any given time. Most processes have a supplier and all have a customer (to whom an output goes), or else the process is useless. What freedom should business entities have to define their product, the enterprise, and the processes they need to produce the product? (Standards issue \#1)

Each product passes through several life-cycle phases from its conception through its disposal. Enterprises themselves pass through these phases. Indeed, some enterprises have products that are projects to design, deliver, and support new enterprises. Manage, design, produce, procure, and support are the life-cycle phases that this paper uses at a high level, just beneath the enterprise level. Enterprises, by way of their goals and strategies, will make conscious decisions about which of these phases receive the most focus for the product they supply. Effective and consistent enterprise models that are consistent will allow parts of different enterprises to combine to provide the complete life cycle for a product; for example, in a virtual enterprise scenario.

An enterprise by the definition, is scalable. It can become somewhat of a challenge to define which processes are intraenterprise and which ones are interenterprise. It really shouldn't matter, if humans or software knows the interfaces between processes, perhaps in conformance with standards. This model is copacetic with the notion of creating and dissolving virtual enterprises on the fly to contribute particular processes to the business of making the overall end product. As long as independent human thought drives the process of enterprise improvement, it seems that enterprise scope will remain a management decision tied to the vision, goals, and strategies. (Standards issue \#1)

Integration refers to a state of enterprise operation in which all necessary things, processes and infrastructure, are in place that enable the communication of correct information, at the correct time, every time. Information flow is at the heart of successful integration. Of course, many other things must happen both technically and culturally to allow satisfactory information flow. What must exist to enable satisfactory information flow and how much of that should be standard? (Standards issue \#2)

\section{ENTERPRISE DESIGN AND ENTERPRISE OPERATION: DRIVERS}

As stated, an enterprise can be any set of related processes we wish to analyze. However, the enterprise has a character and there is some implicit or explicit vision that drives it. Tools help an enterprise accomplish its outputs. Who buys these tools, who selects them, determines if one is better than the other, and decides to invest the money or to wait? How do the tools communicate? How does the enterprise acquire, move, and ship its information and physical things? There is an executive function that decides these things. This is not necessarily a person at the executive level but the function. This function receives its inputs and constraints from a process that determines enterprise goals and strategies and allocates resources that implement the strategies, in an attempt to meet the goals. This process must operate even more smoothly in a virtual enterprise.

If virtual enterprises are to be the mode of enterprise operation for the near term, an enterprise will have to analyze new relationships, and form and dissolve these relationships in relatively short time. There will be processes available for hire, and enterprises shopping to append these processes to their enterprise. Enterprises will have to marry process capability to 
product requirements quickly. This will underline the need to have executable or computersensible enterprise models. Some degree of standardizing the interfaces is necessary to allow virtual enterprises to integrate their operations at the speed and flexibility that is necessary to be a competitive supplier of products or processes. (Standards issue \#3)

There is much technology involved with enterprise operation. To improve the level of integration, certain improvements to technology are necessary. The categories of integration technology divide into infrastructure and process technologies. Infrastructure improvements benefit more than one process in the enterprise whereas a process improvement focuses on one process. If one considers all of the possible infrastructure and process improvements it is not likely that any enterprise will have invested to the maximum in all possible areas. However, the enterprise still operates and it ships products. The improvement areas are, therefore, not a set of specific and fixed hurdles. More accurately the improvement areas are continua, and each enterprise is free to position itself at a particular spot on each continuum. Enterprises base this placement decision on the strategies of the enterprise, the amount of investment money available, and any other criterion important to the enterprise. Also the technology level of each continuum is moving. Is there any value in defining which attributes constitute an integrated enterprise and which do not? (Standards issue \#4)

People or machines analyze, design, simulate, operate enterprises with tools. To justify the investment of the tool builder and to make the tool cost reasonable, the tools must be compatible so that more than one enterprise can use them. The tools must be extensible, migratable, and interoperable so that an enterprise can evaluate a virtual-enterprise opportunity and then hook up to the other enterprise. The other enterprise will be operating at its own chosen points on the various continua. Which components of these transactions should standards constrain: model structure, model content, interfaces between models, and/or known hook-up points so that the software knows with what it dealing? Which standards in place will increase the market for tools, thereby justifying tool-development costs? (Standards issue \#5)

\section{ENTERPRISE-REFERENCE ARCHITECTURE, ENTERPRISE FRAMEWORKS, AND VIEWS OF THE ENTERPRISE}

Definitions for architecture and framework abound. Some definers say that the two words are interchangeable--a serious waste of semantic power. This discussion precedes a set of definitions that will serve as definitions in this paper. The ISO TC184/SC5/WG1, Industrial Automation Systems and Integration, Modeling and Architecture, will attempt to arrive at consensus definitions for these and other enterprise-relevant terms and publish them for more general use.

\subsection{Architecture}

A thing that enterprise analysts, designers, operators, and maintainers need is a reference architecture that they understand to the extent that one enterprise can find a way to use another enterprise architecture, for whatever reason. Users, vendors, and standard makers should investigate and discuss whether standards or software can better enable the required understanding. The things we have with which to work are the enterprises, that comprise tools, material, energy, information, and labor; enterprise-reference architectures; frameworks; enterprise models; suggested or required methodologies; enterprise-semantic elements; and guidelines and rules for using each. The architecture is the organization of all of these things. 
An enterprise-reference architecture should point toward purposeful organization of enterprise concepts. The architecture should have a set of characteristics apropos to enterprise analysis; namely it:

- Adapts to various human and computer-based uses

- Is stable and is it perceived to be stable by users

- Communicates ideas and experience

- Is based on user needs

- Conveys a style that can evolve logically; based on tradition, including the heritage of the past

- Can include contributions of enterprise cultural influence

- Can include logical innovations by the architect

- Is transportable and/or standardizable (even though it may be advisable not to).

The enterprise-reference architecture does not need to have a geometric shape, or orthogonal axes; it could be a document that organizes, logically, detailed knowledge about an enterprise, its purpose, and how it operates.

To be complete an architecture must contain the guidelines and rules for the framework and the remaining structure and systems; a definition of the building-block types; and a definition of available building blocks, or modules (systems), that the object of the architecture should have. When complete the architecture should incorporate the above characteristics.

Definition for this paper: Architecture=Enterprise-Reference Architecture=The body of topical classified knowledge for representing enterprises to design, build, operate, and model them. The architecture contains guidelines and rules for the representation of the enterprise framework, systems, organization, resources, products, and processes.

\subsection{Framework}

The architecture, among other things, defines the nature of the framework--the limiting structure and its supporting members of the instance of the thing being built. A framework is an interlocking set of standards or principles governing behavior, organization, processes, resources, communication, and information. These are principles that give reference, meaning, orientation, or viewpoint to an enterprise and its composite systems. Since an operating enterprise is a dynamic thing a framework should include some kind of system of reference, from which one studies enterprises in operation, in transition or in equilibrium. The framework defines the scope and components of the enterprise under consideration. As with the enterprise-reference architecture, the framework does not need to have a geometric shape, or orthogonal axes; it could be a document or a matrix that defines areas of an enterprise that an analysis should address.

Definition for this paper: Framework=The delineation of the components and viewpoints that comprise a specific enterprise representation and the relationships that exist between the respective viewpoints of the components.

\subsection{Views}

Analyze for a moment what is going on in an enterprise. There are processes building product, many things changing state; people and machines transmitting, receiving, and understanding information; watchers controlling processes; sensors sensing; and electrical power grids and communication grids operating. There are many interconnected systems at work that are 
suboperations of the enterprise. If someone wanted to connect one enterprise with another, or control the factory with a robot, one must control these systems simultaneously. The best form of control depends on the enterprise, and it may not necessarily be hierarchical or centralized but could more closely resemble a chaotic mode of operation.

To achieve an orderly analysis or improvement, there needs to be someplace a representation of these different suboperations or grids. These are commonly referred to as views of the enterprise. There is a finite number of views that if considered, will provide an acceptable computer-sensible representation of the enterprise. (Standards issue \#6)

As stated, the purpose of enterprise integration is to allow timely, repeatable, and accurate information to flow between enterprise processes. To do this requires several technologies and much organizational cooperation and coordination. To be sure that the enterprise model reflects the situation happening in the real world, each set of processes and infrastructure elements under review probably will have to be analyzed from more that one viewpoint; for example, a management view, a technology view, and an information view. (Standards issue \#6) Also, the information collected for the model should describe the state, function, and capability of the subject processes. Computer-sensible modeling of state and function is a developing technology. In an operating scenario this information must be available to some executive, human or machine, responsible for successful operations, so that the executive can make informed decisions about operations.

\subsection{Enterprise Architectures}

If an analyst compared two independently generated enterprise-reference architectures, how similar would they be? If there existed a technique to resolve nomenclature problems, would enterprises be able to understand each other's information? While enterprise designers design what they feel is an optimum way to accomplish the work of the subject enterprise, one enterprise is remarkably similar to another, from the high level down to almost the bottom. Is it then possible, imperative, or desirable that all enterprise structures be the same to achieve enterprise integration? The probability that international standards will constrain enterprise structure is very remote, therefore an attempt to standardize those things would be a waste of time because the standards would be unused?

The body of knowledge that covers all of this, enterprise structure, the enterprise representation framework, rules and guidelines for enterprise representation, the theory of views, and the models, forms the enterprise-reference architecture. Exactly what part of the reference architecture must be standard, and whether or not it matters if there are several different architectures, needs considerable discussion. (Standards issue \#7)

\section{ENTERPRISE MODELS}

Is it possible to determine, in specific and generic ways, what characteristics of an enterprise are necessary to analyze to help achieve an improved degree of enterprise integration? It seems that these would be the key elements of a reference architecture for enterprise creation, operation, and analysis. Once these elements are placed into a logical arrangement necessary for a reference architecture, there would exist an excellent reference architecture for an enterprise model. Therefore one could view an enterprise-reference architecture as a high-level enterprise model or a metamodel for a set of enterprise models. The elements of the reference architecture would be a framework that would indicate the key things in the enterprise that one should consider when creating, analyzing, or using an enterprise model. 
Assume that the most generic, or meta, level for enterprise models is the enterprise-reference architecture because it defines the key aspects to consider to allow enterprise processes and enterprises to interoperate. The reference architecture, using a framework, then defines, among many other things, the required elements of enterprise models. The enterprise can then be modeled downward toward individual activities until the information on the models becomes irrelevant to the task requiring the models. Different tasks may require different levels of models. It is important to model processes consistently for both intraenterprise and interenterprise use. Many analysts feel that the idea of views helps to keep the models consistent. Are views a tool to accomplish this or should specific views be a required feature of an enterprise model? (Standards issue \#6)

The characteristics of effective enterprise models probably are quite specific and fairly straightforward. It seems that here is a good area to constrain the enterprise representation. If we assume that the enterprise is model driven, it seems logical that standards constrain the end products of the representation of components in an enterprise. The logic continues as follows: Innovators will continue to design enterprises by seeking optimum solutions, as discussed in issue \#1. They will continually update and reorganize processes and infrastructure. However, each process or component of the enterprise, both process technology and infrastructure technology, will need the same things to interoperate. This means that when modeling these components, if the information presented in the views is consistently there; say, required by a standard, designers could connect enterprises or pieces of enterprises to other enterprises and operate effectively. Therefore enterprise models appear to be a candidate for standardization. (Standards issue \#7)

\section{BENEFITS OF ENTERPRISE-REPRESENTATION MODELS}

Enterprise models provide a data-driven and model-driven enterprise with several capabilities. Whether or not the integrated enterprise operates in a hierarchical, deterministic mode or in a distributed, chaotic mode, the enterprise model will provide the operator or executive, human or machine, with a map of the enterprise and some knowledge of what functions the enterprise comprises, in what state they are, and what capabilities exist at any moment to accomplish an output. If the models link into some established framework, enterprises can seek, evaluate, set up, and go more easily toward interenterprise as well as intraenterprise commerce.

With well-designed standards about enterprise-representation models in place to provide a known environment to the developer, the risks of investing in an island of integration will be significantly reduced. If confronted with one of those islands, the technology required to interact with a standard environment will be a known quantity.

A level of integration to which enterprises are, or will be, striving is model-driven enterprise. To accomplish this will require computer-sensible models that cause process actions in the factory. To be computer sensible there will be provision in the models to educate the application systems with some knowledge about themselves and their missions. There will be an executive system that is aware of itself and its role for the enterprise. The executive will also be aware of the goals of the enterprise and act to achieve them. Subsystems will also know their roles in the enterprise.

A good standard will guide and constrain existing and emerging enterprise models so that resulting pieces of enterprises will interoperate with each other and formulate migration strategies with confidence. The resultant environment will create a more confident investment climate for integration-technology related human and technical resources.

Enterprise self analysis is driving a voracious appetite for improved manufacturing and information technologies. Needs and requirements for these technologies develop, hopefully, 
using activity, information, dynamic, and behavior modeling. The process of analyzing the enterprise is perhaps the most important reengineering activity because it enables a relatively deep understanding about what is really happening in the enterprise processes. From this understanding, together with the enterprise goals and strategies, come justification for improvements to integration.

\section{ANALYSIS OF THE STANDARDS ISSUES}

\subsection{Standards issue \#1: How much freedom should businesses have to design the enterprise of their choice, with freedom will there still be acceptable integration, and is the idea of a standard enterprise realistic?}

Businesses typically consist of groups, divisions, departments, and sections. Somewhere in that hierarchy the organization becomes function oriented and the higher manager establishes incentives to lower managers to reduce the costs of their function. When that happens there is no advantage to doing things for the benefit of the enterprise because process and infrastructure improvements tend to suboptimize and even conflict with similar investments in other departments. Subsequent reorganization will attempt to fix the problem and the enterprise designers will be busy. This cultural problem will exist whether or not there exist standards recommending otherwise.

For these reasons the standards organizations should recognize that they would waste resources to attempt to standardize enterprise design, or process design, or a hurdle level of integration required to qualify as an integrated enterprise (see Standards Issue \#4). Even if improvements that benefit the entire enterprise were feasible, humans will always demand the freedom to improve enterprise operation through redesign.

Albert Colin of France recommends a standards model that resembles the ISO 9000 series of quality-assurance standards. Rather than setting specific limits to everything, such a standards set would force enterprises to analyze and document, perhaps electronically on line, what they do; and then operate their enterprise accordingly. Lower-level standards would still define interfaces and the supplying process would indicate which ones apply, together with the set of information they present on line about their processes. This way, an enterprise shopping for a process would be able to evaluate, on line, that a process meets both quality and integration guidelines and that they conform to listed interface standards. The shopping enterprise could then advise its software to make any needed adjustments to its enterprise models (function, hardware, software, communication, and information), establish a relationship, and operate in an acceptable (to both parties) level of integration.

\subsection{Standards issue \#2: What must happen to assure proper information flow?}

Proper information has been flowing between processes for hundreds of years--or else manufacturing enterprises would have produced no products. What enterprise integrators are trying to do is to make the process repeatable, accurate, and computer sensible. Knowing which standards are necessary depends on existing standards that are in use; for example, how much of the information at the interface between applications is in an open format, how much of the semantics is in the one-name-one-meaning category, how compatible is the hardware, how well defined are the software interrelationships, are the communication protocols agreed upon, and whether there is understanding about the information architectures. Even with that defined, the business rules must be compatible, government regulations for commerce must be agreeable, and the people of the enterprise must truly want integration. 
What material qualifies for consideration as a standard? The easy, but unrealistic, answer is everything. The appropriate answer involves some tradeoffs. One must consider the rate of technology change versus the ability to create and change standards. And, with standards, one must evaluate what flexibility one surrenders in our ability to migrate to a newer technology that will enable us to achieve a higher degree of integration.

\subsection{Standards issue \#3 Are there special standards required to enable virtual enterprises?}

A virtual enterprise is an enterprise, if we stay with the definition: any set of processes that we wish to analyze. Technically the standards categories involved are the same: hardware, software, communications, information, and architecture. The notion of virtual enterprise brings in a time element such that the enterprise can form or dissolve very quickly. To accomplish this, standards other than just technical ones must be in place. A virtual-enterprise candidate may have to negotiate such contracts as a basic-ordering agreement in advance of considering such an alliance. Collaborating enterprises must resolve other things in a generic sense long before true virtual-enterprise commerce can succeed. Eventual parties to the enterprise will probably pre-define the following, currently often locally unique: financial standards, environmental-impact standards, markup language, safety requirements, statutes, product liability, quality, and organizational requirements.

Perhaps a process similar to the Uniform-Commercial Code in the U.S. will emerge. Perhaps entitled Uniform Virtual-Operations Code (UNIVOC?), it would provide virtual business entities with a consistent and uniform set of business-related standards. Groups similar to those that certify compliance with the ISO 9000 series of quality-assurance standards could administer the process.

\subsection{Standards issue \#4: Is there any value in defining what constitutes an integrated enterprise and what does not?}

Each enterprise is unique with respect to determining how to improve it. Here is where the enterprise character, goals, and strategies come in. The enterprise-executive function selects the level of investment that is appropriate for each process and infrastructure area. These areas appear as continua of opportunity and capability rather than a specific hurdle of capability. Each enterprise will have a self-determined priority for which projects will receive investment and where on the continuum the enterprise will live. Some enterprises will want to be on the leading edge and others may choose to follow the leaders; perhaps to enable them to invest in these technologies later at a lower level. Neither strategy is incorrect, but they lead to unevenly equipped enterprises that are trying to do business in the global marketplace. With well-planned standards, these unevenly equipped enterprises still would be able to interoperate to the degree that their investments permit. A low level of integration capability will not have all of the functionality of the high level. This is sort of like investing in stereo-system components or buying different telephone or cable television capability. It would, therefore, seem useless to specify exactly what must be in place to have enterprise integration.

Defining a specific set of capabilities that would qualify an enterprise as integrated would tend to codify the enterprise strategies and reduce product differentiation. The idea of a standard-capability set is contrary to the operation of a free-enterprise market and not feasible for current and foreseen world-commerce situations. 


\subsection{Standards issue \#5: What should be standard and what should be good software design?}

This issue is difficult because the resolution lies in a range between those opinions that say they need none or few standards and those who say they need to standardize and constrain everything. It is often the easiest and least-effective solution to a computer-compatibility problem to standardize everything. The loss of user delight, the loss of opportunity to use tools especially designed for the job, and the lost opportunity for technological improvement is enormous--and it would stay that way because standards always consume more time to develop than the technology that standards are attempting to constrain. Shortening the time it takes to make standards is not necessarily a good idea. Imagine a world with many easily and hastily constructed standards.

About ten years ago the U.S. Air Force envisioned a design-engineering-tool environment that hinted at a solution that would probably work here. The theory was that there were and will be many tools available to do the many special jobs in product design. There are several options available to get the tools to interoperate and they involve either standardizing the tools, using one tool developer, or finding a way to make different, non-standard tools interoperate through the software in the tools. If one wanted to import a spreadsheet table into a word-processing program, the tools themselves or the operating system would be able to tell from looking at the code what would be necessary to accomplish what the user wanted, and then do it. The only standards would be the basics, such as ASCII and a common nomenclature. At the time this was first discussed it seemed futuristic but now it seems feasible; indeed, it is being done for a U.S. Air Force project called the National Industrial Information Infrastructure Protocol Project, NIIIP.

The NIIIP-mediated architecture is probably the way of the future rather than standardizing everything. Using a music metaphor, mediation lets the software and the knowledgebase say: "Hum a few bars and I will catch on to what's happening." Otherwise we would have to standardize the notes, the rules, the arrangements, the key--everything. The standards will change much more slowly than the technology improves. This would stifle innovation. ISO TC184/SC5/WG1 will analyze the real need for enterprise-integration standards by approaching the topic from a mediated-architecture viewpoint.

Enterprise applications should be able to operate similarly with only a minimum of standards limiting the functionality of tools. The proposal, then, is to look for ways to allow the tools themselves to work with the software in the integrated enterprise and not rely upon standards. To force tool builders to add cost to their products and comply with a set of unnecessary standards may be too limiting, for technological and flexibility reasons.

\subsection{Standards issue \#6: Is there any value in specifying views in standards for enterprise representation?}

To represent the enterprise to the degree that models are executable and the enterprise is model driven will require representing many enterprise phenomena. As the computer-aided design of a complex product divides the complexity into groups of related technology called layers, the enterprise model is sufficiently complex to do the same, and they are generally called views. The enterprise modeler will choose what is important but there are some choices that seem to be applicable generically. The exact number of views depends upon what is the purpose of the models. Generally, there is a view that includes such administrative things as organization, costs, quality assurance, and human interactions--which will be referred to here as a management view. A technology view covers such physical interfaces as hardware interconnections, software hooks, electrical networks, and communication protocols. An 
information view includes the semantics and syntax of the information being exchanged between processes or from process to a logically central database. The database itself and the data-transmission network are in the technology view.

Some companies are in the business of providing an enterprise to a customer. These companies are aware that the enterprise has a life cycle as does any other product. Whenever a customer orders an enterprise there is a need. When the need is combined with customerenterprise goals and strategies, the customer then generates detailed requirements that act as part of the specification for the new enterprise to the enterprise builder.

During the design phase, the enterprise builder creates enterprise models that define the same views that are necessary to operate and support the enterprise later in the enterprise life cycle. In the past, blueprints or engineering drawings were the enterprise models, but they were enterprise models just the same.

When delivering an enterprise, the enterprise designer transfers some of these models to the operator. It was a package of drawings. If the designer made this set of models properly they would be computer sensible and the customer enterprise could then use them to help operate, maintain and dispose of the enterprise. If the models were of highest functionality the supplier and customer could consider the customer goals and strategies, combine them with the enterprise models, and form a system to operate the enterprise automatically. This would be sort of like a white-collar robot that would know who it is and what its job is, take the daily production plan and make decisions based on what happens during a day.

The point is that one set of enterprise models constructed to have the same views can be transferred from the enterprise-design phase, to the operation phase, to the maintenance phase, and used later to do any enterprise reengineering. The customer enterprise or the enterprise builder could do the reengineering with a significant amount of participation by the customer enterprise. The enterprise viewpoint, not necessarily the view names, needs definition and consistency throughout the enterprise life cycle, and the information presented must be rich enough to perform computer-aided-enterprise design, operation, support, and reengineering.

The same logic holds for the virtual-enterprise scenario where two independently developed sets of enterprise and process models will link for a temporary period. Redoing one or both sets of models for that purpose probably is not cost effective. The modelers must achieve some consensus, perhaps in the international-standards arena, with respect to what things they will consider to be important to have in an enterprise or process model.

Each of the major entities in an enterprise is a real thing that ISO TC184 SC5/WG1 calls, for now, a component. Each component will have some or all of these views and subviews applicable to its satisfactory operation in the enterprise. When finished each enterprise model will have several networks represented. For example, for a component called "metal-removing process" there will be a place in the enterprise model that indicates this process and the supporting infrastructure. Included will be several separate model networks that show how to interconnect all of the elements in that particular view and what stuff goes to or from each process. The information model will define the nature of information coming into this process, going out, and what controlling information the process requires. There will be in the hardware view a place on the electrical grid that shows what kind of power goes in and what electric-power conditioning the equipment requires to maintain a successful environment in the factory; and what kinds of connections physical systems require, such as material handling. There will be a model covering what software is in use and how it interfaces with software of other components. There will be a management view that includes what skills operators need. In other words, for each process or component there will be several models, each showing how the elements within cach respective view interconnect. 
Furthermore, from an integration standpoint, it is almost irrelevant whether one is observing an entire enterprise or a single process. The views one must consider probably are the same. Some of them may be null at certain processes; for example, a belt-driven lathe will not have connections to the electrical grid. This is the beginning of a framework, a reference architecture, and an enterprise-modeling scheme. The more accurately these things and ideas can be represented, the more probable it is that there will be a successful implementation achieving integration.

When representing enterprise processes an analyst must consider certain key ideas. Whether it is easier to organize these ideas into views and then collate them or to work with them uncategorized is a matter of preference. Eventually, integrators will need to work with the key ideas, so it seems more important to know what the idea is rather than in what category it is.

6.7 Standards issue \#7: With respect to enterprise representation, what level of concept should be standardized, from entire standard enterprises to standard names of things? Of what value are standards covering enterprise models, enterprise modeling, enterprisereference architectures, or frameworks?

If, as presented in issue \#1, standardized enterprises and processes are not feasible, then at what level is a standard appropriate? The domain consists of hardware, software, communications protocols, information, frameworks, and architectures. There are things, the connections between the things, the information, and the information formats.

Interfaces are a good start. Hardware and communication interface standards are rather mature. Information formats are becoming available with ISO 10303, STEP, or Standard for the Exchange of Product Model Data, and others; and the electronic-data-interchange standards, ANSI X12 and EDIFACT. Architectures and frameworks need to have known or common, but not necessarily standard, interfaces to the extent that when one interfaces with another the pieces of one are mappable to pieces of another. Software interfaces are receiving attention with the CASE Data Interchange Format, CDIF.

One issue that most knowledgeable enterprise integrators agree upon is that terminology is a problem; that is, there needs to be some sort of unambiguous naming dictionary. Possible solutions are the ISO 11179, parts 1-6, Data Element Standard that is under development, the basic-semantic repository (X12 and EDIFACT), and the Universal Data Element Framework being developed by the CALS Enterprise Data Task Group chaired by Ron Schuldt of Lockheed-Martin Denver.

Mr. Schuldt has been espousing the Universal Data Element Format for the last five or six years. Now he is gathering support in the international CALS community to have a centrally registered naming house that would serve to unite the semantics of different naming schemes. $\mathrm{He}$ is not advocating that everyone in the world name things his way, only that they map to his scheme. Two different applications could use any name they want, map to the standard, and thereby link up their semantics.

The basic-semantic repository, BSR, being developed at the ISO Central Secretariat for electronic-data interchange is an approach that can apply to the enterprise-representation domain.

The concept is fine as long as the three, or however many there are, come together soon before there are three or more solutions requiring tons of work to map to each other. Of course STEP would have to participate and cooperate because many of its entities fall in the same tank as the entity names covered by this proposal.

Standardizing the enterprises, parts of the enterprise, the products, the information transferred, and the processes, is probably not going to be a productive use of standard-making 
resources. What seems more usable is to standardize the interfaces, the nomenclature, and the formats and allow the enterprise-tool builders to use these standards to design software in such a way as to allow the processes to communicate.

The enterprise model will allow more consistent modularization so that enterprises can interchange pieces. The models will ameliorate the need to develop the entire system at one time. Simulation will be possible allowing evaluation of interoperation with interenterprise entities and evaluation of systems with differing granularity. Enterprises will be able to plan migration paths more effectively. Because information will be a separate asset, changing applications will be possible without re-entering information about the products and processes unnecessarily. Enterprises can define paths to make the product and process information tie logically into enterprise goals, strategies, capabilities, and business rules. The models should be scalable so that a high-level model is essentially the same as a lower-level model--that is, use the same modeling constructs for all levels.

\section{ISSUE SUMMARY}

Cultural inertia will limit the effectiveness and use of standards to constrain enterprise design. Defining and representing the interfaces that exist in a manner similar to the way the ISO 9000 series of standards does may enable enterprises to communicate without standardizing an enterprise or process structure. The process of evaluating how best to communicate electronically will involve some tradeoffs. The value of standardizing each enabler in the information-integration process must evaluate whether the technology used to achieve acceptable functionality has a life that is shorter than the time it takes to develop and/or modify a standard to constrain the technology. The alternative to standards is to use the technology to circumvent the problem.

A virtual enterprise is not really different from a traditional enterprise other than the fact that it can append and shed processes quickly. There are more legal and regulatory issues than technical issues when removing barriers to virtual-enterprise operations.

Considering the breadth of technology required to integrate enterprises, the speed at which the technology progresses, and the freedom to apply technology that suits, it seems fruitless to create a metric that evaluates whether an integrated enterprise exists or not. An enterprise will have to manage its limited investment resources wisely to be able to communicate in the global-commerce environment at the level that it chooses. Part of this investment will probably involve providing software applications with enough knowledge about the necessary interfaces to mediate the interface connections. Thereby, mediation will achieve satisfactory communication rather than relying on a full set of standards.

There are several areas to evaluate when analyzing enterprise operation. If sets of models from one enterprise are to connect to models of another enterprise, both sets of models must have considered the same areas or the models probably will not interoperate. To ascertain whether modelers can manage these areas consistently informally from a checklist, or formally by standards, needs further implementation experience and discussion to decide. The issue needs satisfactory resolution because the areas in question are precisely the interfaces that one enterprise will mediate with another to communicate.

Areas of integration technology needing standards-like attention are hardware, software, communications protocols, information, and architectures. Of these, enterprise-representation software and enterprise-representation architectures are not yet adequately addressed. There are a few areas that appear to be good candidates for standards. One is terminology and the another is enterprise models. The solution to the terminology problem appears to lie in some registry to which individual enterprise can map its terminology. Enterprise models are a 
product of a technology-driven methodology. If standards constrain enterprise models properly they would reveal the necessary points from which one enterprise architecture can mediate an information transfer with another enterprise architecture.

\section{CONCLUSION}

How can standards help in the enterprise representation process and how can they hurt? What material will standards best constrain and what can well-designed software work around?

Standards must define only what is necessary so that the software developer knows with what the software must work. Ideally, standards should enable interoperability and still protect innovation, efficiency of approach, and migration capability.

Ensuing enterprise representation standards should not mandate standard processes, or standard enterprises, or standard organizations, or standard-enterprise data. These standards should define the key ideas involved with enterprise frameworks or enterprise models so that the models produced are consistent, and so that vendors can develop tools to produce and operate frameworks and models with minimum investment risk and with the maximum confidence that they will encounter known interfaces.

\section{James G. Nell}

Jim Nell is Convener of ISO TC184/SC5/WG1, Automation Systems and Integration, Modeling and Architecture. He is a member of the US delegation to SC5, and a member of the US technical-advisory groups to TC184 and SC5. At NIST, he is the principal investigator of the Manufacturing Enterprise Integration Project to evaluate the use of architectures, frameworks, and enterprise models for use by business entities.

Formerly he was active in the TC 184 SC4 work to develop product- and process-data representation serving as the US expert to the SC4 Strategic Planning Advisory Group. For the IGES/PDES Organization, he was Chairman of the Steering Committee. He was the original chair of the Evolving Standards Focus Group of the Agile Manufacturing Enterprise Forum at the Iacocca Institute, and a founding participant of the ANSI Organization for Harmonization of Product Data Standards. As a member of the National Initiative for Product Data Exchange staff, he was the architect of the NIPDE Electronic Library on the World-Wide Web.

Jim Nell was formerly Manager of Information Technology Programs at the Manufacturing Systems and Technology Center of Westinghouse Electric Corporation in Columbia, Maryland USA. During the 32 years prior to his retirement from Westinghouse, he had various assignments in systems engineering, international marketing, program management, and strategic planning. From 1984-1993, he concentrated on product-information representation, enterprise integration, and standards that apply to information representation and enterprise integration.

He received his BSEE from Drexel University and his MBA from Bowling Green State University. 